\title{
ON THE INFLUENCE OF ATROPIA ON THE HEART.
}

\section{B y Dr. H, G. BE $\mathrm{E} E \mathrm{ER}$, U. S. N.,}

Honorary Curator, Section of Materia Medica, U. S. National Museum.

Last winter, while engaged in studying the physiological action of atropia on the heart, I found among the many terrapins I had occasion to use one whose heart was in a peculiarly abnormal condition. The auricles were very large, larger in fact than the ventricle in diastole, and although contracting in rythmical sequence with relation to the ventricular systole, their contraction was barely perceptible, while the ventricle performed its work in a most satisfactory manner; that is to say, an attemptat contraction on the the part of the auricles was immediately followed by the ventricular systole. I determined, however, to proceed to isolate it in. the usual manner and begin observations on it with atropia.

After the operation of inserting the inflow cannulas into the respective veins, and the outflow cannulas into the arteries, on allowing the nutrient blood-mixture to flow through the heart, no change in its condition was noticed. No lowering of the venous pressure would make the least difference, and even a venous pressure of only $0.5^{\mathrm{cm}}$ was still sufficient to keep the auricles in their distended condition.

Hoping, nevertheless, to induce the auricles to perform their normal work, well oxygenated fresh nutrient blood-mixture was allowed to run through it for over two hours but still the condition remained unaltered.

A back flow of blood from the auricles into the venous cannulas and inflow tubes could be plainly seen to occur with each ventricular systole, showing the undoubted existence of a free and direct communication between ventricle and auricles and auricles and veins. Their respective valves, therefore, were clearly insufficient from the distended condition of the auricles. All the more surprising was the change which occurred when atropized blood was substituted for the normal nutrient blood-mixture, as the following record of the experiment will show.

The heart under observation, being clearly an abnormal one, and therefore not to be found in every terrapin, though it might perhaps be artificially produced without serious injury to the organ, I nevertheless, determined not to publish it until an opportunity offered itselit for verifying the results attained.

I was fortunate enough to open a terrapin last January which presented a heart in a similar, if not identical, condition, and it was at once concluded to try atropia with the result of inducing the inactive auricles to perform their work and thus increase the entire amount of work done by the heart from 100 to 150 per cent.

Inasmuch as these two experiments are strikingly well calculated to throw important light on the stimulating influence exerted by atropin 
on the heart's action, more especially on the auricular portion of it, which was in the two cases in point in a pathological condition of not infrequent occurrence in mammals and man, the publication of at least one of them was deemed justifiable.

For a review of the literature of the subject as well as for a description of my method of experimentation I must refer, in order to avoid repetition, to the studies from the Biol. Lab. Johns Hopk. Univ., vol. iii, No. 2, p. 73, and also to the last number of the Proceedings of the Naval Medical Society.

February 28, 1884. Experiment I. Terrapin 1065. Calf's blood and Ringer's saline 1:1. Cunulas in inferior vena cava, hepatic vein, pulmonary artery and left aorta. Venous pressure $3.5^{\mathrm{cm}}$. Arterial pressure $14^{\mathrm{cm}}$. Atropized blood $0.0025: 100^{\mathrm{cc}}$ of blood-mixture. During recovery the heart, being found specially delicate, low pressures have been used.

\begin{tabular}{|c|c|c|c|c|}
\hline 'Time. & $\begin{array}{l}\text { Rate per } \\
\text { minute. }\end{array}$ & $\begin{array}{l}\text { Work in } \\
\text { c. c. per } \\
\text { minute. }\end{array}$ & $\begin{array}{l}\text { Temper- } \\
\text { ature. }\end{array}$ & $\begin{array}{l}\text { The cireulating fluids were supplied to the heart at the time } \\
\text { mentioned on the same line in column one. }\end{array}$ \\
\hline 4. 10 & 35 & 14 & 19 & \\
\hline 11 & & & & On atropized blood. \\
\hline $\begin{array}{l}.13 \\
.16\end{array}$ & 33 & 18 & & On normal blood-mixture. \\
\hline .19 & & ............ & & On atropized blood. \\
\hline .20 & 22 & $\cdots \cdots \cdots$ & & On normal blood-mixture. \\
\hline .25 & 31 & 21.5 & $\cdots$ & $\begin{array}{l}\text { Reappearance of previous condition, viz, both auricles much } \\
\text { distended, left incompletely ; right not at all contracting. }\end{array}$ \\
\hline .30 & 31 & 19 & 19 & $\begin{array}{l}\text { Ventricles and auricles contracting simultaneously. } \\
\text { On atropized blood. }\end{array}$ \\
\hline .32 & & & & $\begin{array}{l}\text { On atropized blood. } \\
\text { Normal blood. }\end{array}$ \\
\hline .35 & 31 & 24 & & Both auricles contracting with much force. \\
\hline .40 & 31 & 23 & & A perceptibly longer interval between anricular and ventricu- \\
\hline .41 & & & & $\begin{array}{l}\text { On atropized blood. } \\
\text { On normal blood-mixture. }\end{array}$ \\
\hline 43 & 21 & 28 & & $\begin{array}{l}\text { Both auricles contracting twice to ventricle once. Ventricle } \\
\text { slightly larger than before, but contracting thoroughly. }\end{array}$ \\
\hline .48 & 16 & 35 & & Ventricle suddenly becomes smaller again. A uricle distended \\
\hline $\begin{array}{l}.53 \\
.543\end{array}$ & 32 & 12 & \begin{tabular}{r|r}
18.5 \\
$\ldots \ldots \ldots .$.
\end{tabular} & $\begin{array}{l}\text { as before, auricular and ventricular systoles simultaneous. } \\
\text { On atropized blood. }\end{array}$ \\
\hline .56 & 17 & 35 & & $\begin{array}{l}\text { Off atropized blood, } 31^{c c} \text { through on } \mathrm{I} \text { blood. } \\
\text { Both auricles again contracting. Ventricle expanding and }\end{array}$ \\
\hline 5.00 & 17 & 35 & ......... & firmly contracting; two auricular to one ventricular beat. \\
\hline .04 & $\begin{array}{l}17 \\
32\end{array}$ & $\begin{array}{l}35 \\
14\end{array}$ & $\cdots \cdots$ & Immediately after this observation was taken, ventricle re- \\
\hline $\begin{array}{l}.07 \\
.09\end{array}$ & 32 & 14 & & $\begin{array}{l}\text { sumed its rapid rate. Auricles became distended as before. } \\
\text { On atropized blood. }\end{array}$ \\
\hline .11 & & & $\cdots$ & $\begin{array}{l}\text { On normal blood. } \\
\text { Ond }\end{array}$ \\
\hline & & & $\cdots$ & A uricles again contracting. Ventricles dilating fully. \\
\hline $\begin{array}{l}.16 \\
.21\end{array}$ & $\begin{array}{l}16 \\
16\end{array}$ & $\begin{array}{l}33 \\
33\end{array}$ & $\begin{array}{r}18.5 \\
\ldots \ldots+\cdots\end{array}$ & \\
\hline .24 & & & & $\begin{array}{l}\text { Sudden return to former condition, viz, auricle distended, left } \\
\text { scarcely at all contracting; right not at all. Ventricle half } \\
\text { the size of auricle; auricular and ventricular systoles simul. } \\
\text { taneous. }\end{array}$ \\
\hline $\begin{array}{l}.25 \\
.27 \\
.293\end{array}$ & & 13.5 & $\begin{aligned} 18.5 \\
\ldots \ldots\end{aligned}$ & $\begin{array}{l}\text { taneous. } \\
\text { On atropized blood. }\end{array}$ \\
\hline .293 & 16 & & & On normal blood-n \\
\hline .37 & 16 & 36 & & $\begin{array}{l}\text { Ventricle largely dilating and slowly, but firmly, contracting. } \\
\text { Left auricle contracting twice to right auricle and ventricle }\end{array}$ \\
\hline .40 & 15 & 35 & & $\begin{array}{l}\text { once. } \\
\text { Sudden return to former condition. }\end{array}$ \\
\hline $\begin{array}{l}.45 \\
.50\end{array}$ & 31 & 11.5 & $\cdots$ & \\
\hline .51 & $\cdots$ & & $\cdots$ & On atropized blood. \\
\hline .54 & & & & On normal blood-mixture. \\
\hline $\begin{array}{r}.57 \\
6.01\end{array}$ & $\begin{array}{l}16 \\
16\end{array}$ & $\begin{array}{l}35 \\
36\end{array}$ & \begin{tabular}{r|r}
18.5 \\
$\ldots \ldots \ldots$
\end{tabular} & $\begin{array}{l}\text { Condition of heart identical with what has been described as } \\
\text { occurring under atropia. }\end{array}$ \\
\hline .05 & 16 & 34 & $\ldots$ & \\
\hline 10 & 16 & 33 & 18.5 & \\
\hline
\end{tabular}




\begin{tabular}{|c|c|c|c|c|}
\hline Time. & $\begin{array}{l}\text { Rate per } \\
\text { minute. }\end{array}$ & $\begin{array}{l}\text { Work in } \\
\text { c. c. per } \\
\text { minute. }\end{array}$ & $\begin{array}{l}\text { Temper- } \\
\text { ature. }\end{array}$ & Remarks. \\
\hline 6.113 & & & & $\begin{array}{l}\text { Abruptly passed into normal condition, with only an occa- } \\
\text { sional expansion and subsequent strong contraction. Auri- }\end{array}$ \\
\hline .13 & 31 & 13.5 & & $\begin{array}{l}\text { cle twice the size of ventricle. } \\
\text { On atropized blood. }\end{array}$ \\
\hline $\begin{array}{l}.143 \\
.173\end{array}$ & & & & On normal blood.mixtmre. \\
\hline .20 & 14 & 29 & is j & On normal blood-mixture. \\
\hline .25 & $\begin{array}{l}14 \\
14\end{array}$ & 30.2 & ….... & Condition of heart identical with what has been described \\
\hline .35 & 14 & $\begin{array}{l}31.5 \\
32\end{array}$ & …....... & under atropia. \\
\hline .413 & 14 & 29 & ........ & $\begin{array}{l}\text { At the end of this observation passed back into normal con- } \\
\text { dition; an occasional full expansion, followed by a long and }\end{array}$ \\
\hline .46 & $\begin{array}{l}26 \\
25\end{array}$ & $\begin{array}{l}16.5 \\
19\end{array}$ & & $\begin{array}{l}\text { strong contraction. } \\
\text { During this observation four strong contractions occurred. }\end{array}$ \\
\hline .59 & 26 & 13. 5 & & $\begin{array}{l}\text { During this observation four strong contractions occurred. } \\
\text { Auricles largely distended. Ventricles small; not coutract. }\end{array}$ \\
\hline 7. 09 & 25 & 13.5 & 18 & ing as rapidiy as betore under normal biood. \\
\hline .15 & 24 & 14 & n......... & On atropized blood. \\
\hline .40 & 16 & 32.5 & $\ldots$ & $\begin{array}{l}\text { un normal atropized blood; ventricle slower than it did before; } \\
\text { interval between auricular and ventricular sy stoles 'ong.r } \\
\text { than in previous observations. }\end{array}$ \\
\hline .43 & 17 & & ... & \\
\hline .47 & $\begin{array}{l}19 \\
17\end{array}$ & $\begin{array}{l}27 \\
25\end{array}$ & & Ventricle contracting oftener than auricles, whicb are slightly \\
\hline .57 & 18 & 28 & 18 & $\begin{array}{l}\text { Ventricle contracting oftener than auricles, whicb are slightly } \\
\text { distended. }\end{array}$ \\
\hline $\begin{array}{r}8.00 \\
.04\end{array}$ & $\begin{array}{l}18 \\
18\end{array}$ & $\begin{array}{l}35 \\
30.5\end{array}$ & …....... & \\
\hline .04 & $\begin{array}{l}18 \\
15\end{array}$ & $\begin{array}{l}30.5 \\
27.5\end{array}$ & ........... & Heart working we!! in evers respect; both auricles doing well, \\
\hline .15 & 15 & 32 & ......... & $\begin{array}{l}\text { Heart working we!? in evers respect; both auricles doing well, } \\
\text { expanding and contracting perfectly; ventricle same; ven- }\end{array}$ \\
\hline .20 & 16 & 33 & $\cdots \ldots \ldots$ & tricular sy=tole following anricular in good order. \\
\hline .31 & 16 & $\begin{array}{ll}35.5 \\
35\end{array}$ & .......... & \\
\hline .40 & $\begin{array}{l}16 \\
16\end{array}$ & $\begin{array}{l}35 \\
34.5\end{array}$ & (n........ & \\
\hline .45 & 16 & 34.5 & ......... & \\
\hline .50 & 16 & 31.5 & $\cdots$ & \\
\hline .55 & 14 & $\begin{array}{l}31.5 \\
33.5\end{array}$ & $\cdots$ & 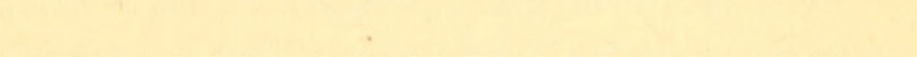 \\
\hline $\begin{array}{r}9.00 \\
.05\end{array}$ & $\begin{array}{l}15 \\
15\end{array}$ & $\begin{array}{l}33.5 \\
29\end{array}$ & $\cdots \cdots$ & Right auricle beginning to fall back, not emptying itself com- \\
\hline .14 & 14 & 35. 2 & & pletely. \\
\hline .20 & $\begin{array}{l}12 \\
12\end{array}$ & $\begin{array}{l}31 \\
31\end{array}$ & $\cdots$ & 1 \\
\hline .30 & 10 & 27.5 & ...... & \\
\hline .35 & 8 & 23. 2 & 18 & Heart getting exhausted. Ended experiment. \\
\hline
\end{tabular}

\section{EXPLANATION OF PLATE I.}

Fig. 1 represents the tracings taken while the heart was working under the influence of atropized blood.

Fig. 2 shows tracings obtained while the heart was under the influence of normal nutrient blood-mixture; it is also intended to illustrate the promptness and complete. ness with which the beart passed out from under the influence of atropia, as can be seen on the left-hand side of the figure where part of the tracing taken under atropized blood is left on for the purpose of showing this, 


\section{$2 \mathrm{BHL}$ Biodiversity Heritage Library}

Beyer, Henry G. 1885. "On the influence of atropia on the heart." Proceedings of the United States National Museum 8(498), 101-103. https://doi.org/10.5479/si.00963801.8-498.101.

View This Item Online: $\underline{\text { https://www.biodiversitylibrary.org/item/52770 }}$

DOI: https://doi.org/10.5479/si.00963801.8-498.101

Permalink: https://www.biodiversitylibrary.org/partpdf/50973

\section{Holding Institution}

Smithsonian Libraries

\section{Sponsored by}

Smithsonian

\section{Copyright \& Reuse}

Copyright Status: Public domain. The BHL considers that this work is no longer under copyright protection.

This document was created from content at the Biodiversity Heritage Library, the world's largest open access digital library for biodiversity literature and archives. Visit BHL at https://www.biodiversitylibrary.org. 